\title{
Aplicación de la técnica de PCR en la detección de Ralstonia solanacearum en campos paperos
}

\section{PCR applied to the detection of Ralstonia solanacerum in potato fields}

\author{
Sanabria, Analía ${ }^{(1)}$, Siri, María Inés ${ }^{(1)}$, Cecchetto, Gianna ${ }^{(2)}$, Pianzzola, María Julia ${ }^{(1)}$ \\ (1) Cátedra de Microbiología, Departamento de Biociencias, Facultad de Química, Universidad de la República, Uruguay \\ (2) Microbiología, Departamento de Química Biológica, Facultad de Ciencias, Universidad de la República, Uruguay. \\ Contacto: mpianzzo@fq.edu.uy \\ Recibido: 15/6/2012 - Aprobado: 1/10/2012
}

\section{Resumen}

La murchera de la papa causada por Ralstonia solanacearum es una amenaza permanente para el cultivo con consecuencias devastadoras en condiciones predisponentes. La incidencia de esta enfermedad en Uruguay es variable; desde la década de 1970 se registran brotes agudos periódicos difíciles de prevenir y controlar. Se ha detectado a $R$. solanacearum en todas las regiones de producción, lo que dificulta el mantenimiento de áreas libres de patógeno y la continuidad del cultivo. Dado que no existen agentes químicos efectivos, las estrategias de control apuntan a la prevención, recomendando la rotación de cultivos, la desinfección de herramientas y el uso de semilla certificada libre del patógeno. En este trabajo se optimizó la detección molecular de R. solanacearum en suelo mediante PCR con el objetivo de conocer la persistencia del patógeno en el campo. El método se aplicó al análisis de cuatro campos con diferentes antecedentes de murchera, y se demostró que el patógeno es capaz de permanecer viable por más de 10 años. También se determinaron las ventajas y limitaciones de esta metodología en su aplicación a muestras complejas. Estos resultados constituyen un importante aporte que genera conocimiento que permitirá a los productores delinear mejores estrategias para el manejo de la enfermedad al momento de planificar los cultivos.

Palabras clave: Murchera, Solanum tuberosum, persistencia.

\begin{abstract}
$\underline{\text { Abstract }}$
Bacterial wilt, caused by Ralstonia solanacearum, is a permanent threat for crops and under favorable conditions its consequences are devastating. The incidence of the disease is variable in Uruguay; since the seventies there have been several hard to prevent and control episodes. R. solanaearum has been detected in all the productive areas. It is a challenge to maintain continuous production and fields free from the pathogen. Since no effective chemistry agents against R. solanacearum exist, the available pest control strategies are crop rotation, disinfection of tools and use of certificated pathogen-free seed potato. In this work the detection of R. solanacearum in soil was optimized using PCR to identify how long the pathogen persists. The strategy was applied to four potato fields with different backgrounds, showing that the pathogen is able to stay viable for more than 10 years. The advantages and limitations of this method on complex samples were determined. These results help potato producers improve pest control management of the crop. Keywords: Bacterial wilt, Solanum tuberosum, persistence.
\end{abstract}

\section{Introducción}

En 1896, Erwin Frink Smith estableció a Ralstonia solanacearum como el agente causal del marchitamiento bacteriano de la papa, el tomate y la berenjena. Este microorganismo está presente en todos los continentes y tiene la capacidad de enfermar a una gran cantidad de cultivos de importancia económica y nutricional, por lo que se considera que es una de las bacterias fitopatógenas de mayor impacto económico en el mundo (Schell, 2000).

En Uruguay afecta particularmente a la papa, uno de nuestros principales cultivos hortofrutícolas. La enfermedad, también conocida como pudrición parda o murchera, produce daños sobre el follaje y los tubérculos y es la segunda enfermedad de importancia mundial en el cultivo de papa. Desde el punto de vista taxonómico, $R$. solanacearum es un bacilo Gram negativo aerobio, perteneciente a la subdivisión $\beta$ de la clase Proteobacteria. Se considera que las cepas de $R$. solanacearum constituyen un complejo de especies altamente heterogéneo que agrupa cepas que varían en virulencia, rango de hospedero, modos de transmisión y propiedades metabólicas. Las cepas presentes en nuestro país pertenecen a la raza 3 biovar 2, según el esquema clásico de clasificación, lo que se corresponde con el filotipo IIB1, según la clasificación más reciente (Fegan y Prior, 2005).

El primer brote de murchera en Uruguay fue registrado en 1974 y su impacto fue devastador. Un segundo episodio de importancia ocurrió en la zafra 2001-2002, cuando el $39 \%$ de los predios sembrados en las tres zonas de producción principales (Sur, Este y Norte) fueron afectados, con mayor severidad en las zonas Este y Norte (MGAPDIEA, 2003). Actualmente, la incidencia de la enfermedad es variable y muy dependiente de las condiciones ambientales y prácticas de cultivo.

Las vías de diseminación más frecuentes de $R$. solanacearum 
son el tubérculo-semilla infectado, el agua de lluvia y riego, así como también la maquinaria, herramientas y fuentes de agua superficiales contaminadas. El hecho de que esta bacteria tenga un gran rango de hospederos y una amplia distribución geográfica limita la implementación de estrategias efectivas de control. Estas apuntan más que nada a la prevención y recomiendan la rotación de cultivos en campos infectados, el uso de material vegetal libre del patógeno y la limpieza de las herramientas utilizadas, en tanto se busca generar cultivares resistentes (Saddler, 2005). Hasta el momento y pese a los esfuerzos que se han realizado desde hace muchos años, no se han logrado medidas de control completamente efectivas. Esto se ve agravado por el hecho de que una vez introducido en el suelo el patógeno puede sobrevivir por varios años, constituyendo un reservorio de la enfermedad difícil de detectar y erradicar (Hayward, 1991).

Varios estudios se han enfocado a determinar la persistencia de $R$. solanacearum en el suelo. Sin embargo, los resultados obtenidos son variables y las evidencias apuntan a que la capacidad de sobrevivencia del patógeno depende de múltiples factores tanto bióticos como abióticos, entre los que se destacan la humedad del suelo, la temperatura, la competencia con la microflora acompañante y la presencia de malezas que actúan como hospederos asintomáticos, manteniendo el inóculo en el suelo a nivel de la rizósfera (Granada y Sequeira, 1983; Elphinstone, 1996; van Elsas et al., 2000; Moffett et al., 2002; Denny, 2006).

Dadas las pérdidas severas que produce, la murchera de la papa se maneja hasta el presente como una enfermedad de exclusión, de modo que la presencia del patógeno inhabilita el uso de una chacra por un período de años. Si bien la rotación de cultivos es efectiva, se desconoce el tiempo de supervivencia del patógeno en suelo en las condiciones particulares de Uruguay. Nuestra reglamentación fitosanitaria establece la tolerancia cero para $R$. solanacearum en tubérculo-semilla de papa. No obstante, esto no es suficiente para asegurar el éxito en la producción, ya que el cultivo debe acompañarse de suelos libres del patógeno (MGAP, 2010). Esto es aún más importante para quienes además desean producir papa-semilla, debido al riesgo de diseminación de la enfermedad en cultivos posteriores.

Una de las principales dificultades para el estudio de la sobrevivencia de $R$. solanacearum en el suelo es la detección del patógeno en una matriz tan compleja y en la cual generalmente se encuentra en un muy bajo número. El surgimiento de la técnica de la reacción en cadena de la polimerasa (PCR) ha permitido desarrollar métodos de detección sensibles, rápidos y específicos que han sido aplicados a la detección de este patógeno en diferentes tipos de muestras complejas, como tubérculos, plantas, suelo, agua, y que contribuyen a su diseminación.

Buscando responder a las necesidades de los productores de conocer la persistencia de este fitopatógeno en suelos paperos, nuestro grupo trabaja desde hace algunos años en la detección del patógeno en muestras de suelos de chacras de Uruguay. En trabajos previos hemos optimizado un método molecular de detección basado en la incorporación de una etapa de enriquecimiento selectivo de las muestras de tierra previo a la amplificación por PCR. El patógeno fue detectado en campos de las distintas zonas paperas, con diferentes antecedentes de murchera y bajo distintas condiciones (Siri et al., 2005; Sanabria et al., 2007). En este trabajo hemos continuado el estudio de persistencia de $R$. solanacearum sobre campos con diferentes antecedentes de murchera, incorporando un nuevo diseño de recolección de muestras. Se presentan los resultados de persistencia del patógeno en campos con antecedentes de infección de 1, 3, 12, 15 y 33 años.

\section{Materiales y Métodos}

\section{Cepa bacteriana y condiciones de crecimiento}

Se empleó la cepa de $R$. solanacearum UY041 (raza 3, biovar 2) como control positivo. Esta cepa fue aislada en Uruguay en 2004 a partir de un tubérculo con síntomas de murchera y pertenece a la colección de cepas de la Cátedra de Microbiología de la Facultad de Química de la Universidad de la República (Siri et al., 2011). La propagación de esta cepa se realizó por cultivo en medio sólido TZC (Kelman, 1954) durante $48 \mathrm{~h}$ a $28^{\circ} \mathrm{C}$.

\section{Muestreo de los campos}

Se evaluaron cuatro campos con diferentes antecedentes de murchera ubicados en las zonas Norte y Este de producción papera (Tabla 1). Los campos I, II y IV tuvieron un foco de infección 1, 3 y 33 años antes de la fecha de muestreo. El campo III fue evaluado en dos oportunidades, a los 12 y 15 años después del foco.

Ninguno de los campos estudiados fueron utilizados para cultivos susceptibles después de la infección con $R$. solanacearum y, en cambio, fueron destinados para pasturas o para producción de trigo, girasol y maíz. En cada campo se tomaron muestras de tierra en 25 puntos distribuidos al azar en todo el terreno, y se registró mediante GPS la ubicación exacta del mismo. En cada punto de muestreo se extrajeron dos muestras de tierra de aproximadamente $500 \mathrm{~g}$ que se mezclaron y homogeneizaron antes del análisis. También se tomaron muestras de tierra fuera del área de cultivo, a los efectos de considerarlas como controles negativos. Las muestras se mantuvieron en bolsas cerradas a temperatura ambiente y se analizaron antes de las $24 \mathrm{~h}$ de extraídas.

\section{Enriquecimiento de las muestras de suelo}

Se inoculó $1 \mathrm{~g}$ de cada muestra de tierra homogeneizada en 30 $\mathrm{mL}$ de caldo selectivo SMSA modificado (Englebretch, 1994). Para cada campo analizado se incluyó un control positivo que consistió en la muestra de tierra tomada fuera del área de cultivo inoculada con $R$. solanacearum a partir de una colonia crecida en medio TZC. Los enriquecimientos se incubaron por $48 \mathrm{~h}$ a $28{ }^{\circ} \mathrm{C}$ con agitación $(150$ rpm).

\section{Detección molecular a partir de las muestras de suelo enriquecidas}

Se realizó la detección mediante PCR a partir de las muestras de suelo enriquecidas en caldo selectivo SMSA modificado. Se tomó una alícuota de cada enriquecimiento y se incubó a $99^{\circ} \mathrm{C}$ durante 30 minutos para provocar la lisis celular y la liberación del ADN. Para la amplificación se utilizaron los primers PS1-PS2 específicos para la especie $R$. solanacearum dirigidos a una porción del gen del $\mathrm{ARNr}$ 16S (Pastrik y Maiss, 2000), siguiendo el procedimiento descrito por Seal et al. (1999). La reacción se realizó en un volumen total de $25 \mu 1$, con 2,5 $\mu 1$ de tampón de PCR 10X (suministrado con la Taq), 0,5 U de Taq polimerasa, $\mathrm{MgCl}_{2} 1,5 \mathrm{mM}$, dNTPs $0,2 \mathrm{mM}, 12,5$ pmoles de cada primer y $5 \mu \mathrm{l}$ de cultivo lisado. Se utilizó el siguiente programa de temperaturas: desnaturalización inicial a $96{ }^{\circ} \mathrm{C}$ por 2 minutos, seguida de 35 ciclos repetidos de desnaturalización $\left(94^{\circ} \mathrm{C}, 20\right.$ segundos), hibridación de los primers $\left(67^{\circ} \mathrm{C}, 25\right.$ segundos), extensión $\left(72{ }^{\circ} \mathrm{C}, 30\right.$ segundos) y un ciclo de extensión final a $72{ }^{\circ} \mathrm{C}$ por 5 minutos. Cada muestra se analizó por duplicado. El tamaño del fragmento esperado es de $550 \mathrm{pb}$. 
Como control positivo de la amplificación se utilizó ADN extraído a partir de una cepa de $R$. Solanacearum, siguiendo los procedimientos descriptos en Sambrook y Rusell (2001). Los productos de amplificación obtenidos se visualizaron mediante electroforesis en geles de agarosa $0,8 \%$ con tinción con bromuro de etidio y se determinaron los tamaños de los fragmentos por comparación con un marcador de peso molecular $1 \mathrm{~kb}$ Smart Ladder (Eurogentec, California, Estados Unidos).

\section{Detección directa a partir de muestras de suelo}

Se realizó la detección directa sobre el ADN total extraído de las muestras de suelo con el kit comercial Power Soil DNA, MoBio Laboratories (California, Estados Unidos). Posteriormente se realizó la amplificación por PCR del ADN extraído, empleando los primers específicos PS1-PS2, en las condiciones descritas anteriormente. De igual forma, los productos de amplificación obtenidos se visualizaron mediante electroforesis en geles de agarosa $0,8 \%$ con tinción con bromuro de etidio y se determinaron los tamaños de los fragmentos por comparación con un marcador de peso molecular comercial.

\section{Secuenciación de los productos de amplificación}

Los productos de amplificación seleccionados se enviaron a purificar y secuenciar a Macrogen (Korea) y las secuencias fueron comparadas con la base de datos Genbank con el software nBLAST (Dumontier y Hogue, 2002).

\section{Resultados}

Se estudiaron cuatro campos con distintos antecedentes de murchera en los que se recolectaron 25 muestras por campo y al menos una muestra tomada fuera del área de cultivo. No se detectó la presencia de $R$. solanacearum en ninguno de los controles negativos analizados.
En la Tabla 1 se muestran los campos analizados y el número de muestras que resultaron positivas en la reacción de PCR luego de 48 $\mathrm{h}$ de enriquecimiento selectivo. En la Figura 1 se representan a modo de ejemplo dos de los campos (II y IIIa), de forma de visualizar la distribución de los puntos de muestreo. También se señala la ubicación de las muestras que resultaron positivas. Se detectó la presencia de $R$. solanacearum en tres de los campos analizados. Para el campo IV, con un antecedente de murchera en 1974, ninguno de los enriquecimientos dio positivo. El mayor número de muestras positivas se detectó en el campo con antecedentes más recientes de infección por murchera (campo I). En este caso se constató la presencia de $R$. solanacearum en 18 de las 25 muestras analizadas. Por otra parte, para el campo II que presentó un foco de murchera tres años antes del muestreo, se obtuvo una menor proporción de muestras positivas (6/25). El campo III fue muestreado en dos ocasiones con diferencia de tres años. El segundo muestreo mostró una disminución del número de muestras positivas.

Como otra forma de demostrar estos resultados, se realizó la detección directa de $R$. solanacearum sobre el ADN extraído a partir de las muestras de suelo. Este análisis se llevó a cabo sobre un número representativo de muestras. Para aquellos campos que tuvieron varios enriquecimientos positivos (I, II y III) se escogieron algunos puntos al azar (4 o 5 muestras) y se extrajo ADN total de tierra. Sobre este ADN se realizó la PCR con los primers PS1-PS2. Excepto para dos de los puntos analizados (campo II, punto 7 y campo IIIa, punto 14) todos los resultados de la PCR sobre el ADN total de tierra fueron coincidentes con los resultados de la PCR sobre el enriquecimiento (Figura 1). Para el campo IV, en el que ninguna muestra enriquecida fue positiva, se realizó la detección directa sobre 10 de las muestras del suelo y se obtuvieron resultados positivos de la PCR para tres de los puntos.

Por último, se analizaron las secuencias de 10 productos de amplificación tomados al azar correspondiendo al género Ralstonia. Debido a que los porcentajes de identidad respecto a otras secuencias depositadas están por debajo del 98,5\%, no se pudo asignar la especie a ninguna de las muestras analizadas (CLSI, 2008).

\begin{tabular}{|c|c|c|c|c|c|}
\hline Campo & Departamento (*) & Antecedente de murchera & Muestreo & Área muestreada (**) & Muestras positivas (**) \\
\hline I & Rocha (Este) & 2008 & 2009 & Nd $(* * *)$ & 18 \\
\hline II & Rocha (Este) & 2003 & 2006 & 33 & 6 \\
\hline III $_{\mathbf{a}}$ & Rocha (Este) & 1994 & 2006 & 20 & 14 \\
\hline III $_{\mathbf{b}}$ & Rocha (Este) & 1994 & 2009 & 20 & 8 \\
\hline IV & Tacuarembó (Norte) & 1974 & 2007 & 20 & 0 \\
\hline
\end{tabular}

Tabla 1. Detección de $R$. solanacearum en campos con distintos antecedentes de murchera.

${ }^{*}$ ) entre paréntesis se indica la zona de producción papera

$(* *)$ en hectáreas

$(* * \star)$ nd, no determinado

$\left({ }^{* *}\right)$ resultado de la amplificación por PCR a partir de los enriquecimientos a las $48 \mathrm{~h}$, sobre 25 muestras totales 
A.

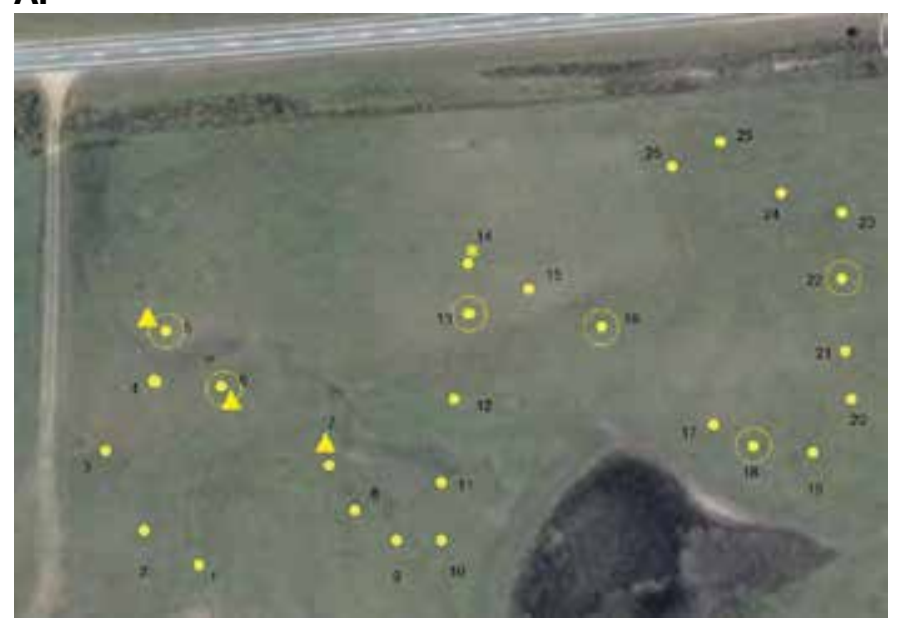

B. de enriquecimiento previo a la detección por PCR también disminuye el efecto de sustancias frecuentemente presentes en el suelo, que afectan la actividad de la ADN polimerasa inhibiendo la amplificación (Picard et al., 1992; Pradhanang et al., 2000; Lin et al., 2009).

La aplicación de este método para la detección de $R$. solanacearum en muestras de suelo permitió detectar la presencia del patógeno en todos los campos analizados con antecedentes de 1, 3, 12 y 15 años de infección por murchera. Como era de esperar, el mayor número de muestras positivas se detectó en el campo con antecedentes más recientes de infección. Sin embargo, no se observó una correlación clara entre la presencia de $R$. solanacearum en el campo y el tiempo transcurrido a partir del foco. Este hecho puede atribuirse a diferencias en severidad de los brotes ocurridos en los distintos campos, así como a múltiples factores bióticos y abióticos que pueden afectar la sobrevivencia de $R$. solanacearum en el campo (Álvarez, 2010).

En el caso del campo muestreado en dos oportunidades (campo III), fue posible correlacionar las muestras positivas con la severidad del foco en esos lugares gracias a datos aportados por los productores. No se contaba con esta información para el resto de los campos. Un factor que puede contribuir en forma importante a mantener la bacteria en los campos es la presencia de malezas capaces de albergar a la bacteria a nivel de la rizósfera, potenciando la sobrevivencia del patógeno en el campo (Janse et al., 2004).

Asimismo, se efectuó la detección directa de $R$. solanacearum mediante amplificación por PCR sobre el ADN extraído a partir de las muestras de suelo sin enriquecer. Esta alternativa se ensayó para algunas de las muestras con el objetivo de corroborar los resultados obtenidos por amplificación sobre los enriquecimientos. La mayoría de las muestras analizadas mostraron una concordancia en los resultados obtenidos por ambas metodologías. Las principales variaciones se encontraron para las muestras correspondientes al campo con un antecedente de murchera de 33 años (campo IV). En este caso no se detectó la presencia de $R$. solanacearum en ninguna de las 25 muestras evaluadas mediante enriquecimiento y posterior amplificación por PCR. No obstante, tres de las 10 muestras evaluadas mediante amplificación a partir del ADN extraído de suelo para este campo resultaron positivas. A partir de la extracción de ADN total de suelo, también amplificaron dos muestras correspondientes a los campos II y IIIa (muestra 7 del campo II y muestra 14 del campo IIIa, Figura 1), las cuales habían resultado negativas por amplificación a partir del enriquecimiento. Estos resultados indicarían la posible presencia del patógeno en un estado latente conocido como "viable pero no cultivable". Se ha comprobado que muchos microorganismos que habitan en el suelo son capaces de adoptar este estado fisiológico en el que la bacteria se mantiene viable pero no es capaz de multiplicarse activamente en un medio de cultivo (McDougald et al., 1998; Alexander et al., 1999). Además se ha comprobado que $R$. solanacearum es capaz de adoptar este estado de latencia que le permite sobrevivir a condiciones de estrés frecuentes en el suelo, como la deshidratación, limitación de nutrientes y exposición a metales pesados (Grey y Steck, 2001; Álvarez et al., 2010).

Los resultados obtenidos en este trabajo indican que $R$. solanacaearum es capaz de persistir viable en el suelo por más de 10 años después de la infección. Según bibliografía, la persistencia de la bacteria en el suelo es variable y va desde varios meses hasta más de cuatro años (van Elsas et al., 2000). Su sobrevivencia depende de muchos factores, dentro de ellos los climáticos, pero también el tipo de suelo y la humedad, entre otros. Al tratarse de una enfermedad de exclusión, para el productor se hace imprescindible conocer la capacidad de sobrevivencia del patógeno en sus campos para la programación de las siembras. De acuerdo a nuestros resultados, el 
tiempo de persistencia de $R$. solanacearum en los suelos analizados superaría el tiempo aconsejado para la interrupción del cultivo de papa luego de una infección (de seis años; F. Vilaró, comunicación personal). Este hallazgo es de suma importancia, ya que repercute directamente en las prácticas de cultivo a adoptar para evitar la reincidencia de esta enfermedad.

\section{Conclusiones}

Mediante la aplicación de métodos moleculares de detección se estableció que $R$. solanacearum es capaz de persistir hasta 15 años luego de la aparición de la enfermedad en el cultivo para los campos y condiciones estudiadas.

El enriquecimiento en medio selectivo con incubación de 48 h y posterior amplificación por PCR resultó un método apropiado para la detección de $R$. solanacearum en muestras de suelo. El método de recolección de las muestras al azar en toda el área del campo se ajusta mejor a campos paperos de varias hectáreas, como lo son frecuentemente en Uruguay. Dado que la bacteria puede estar presente en hospederos asintomáticos, se hace también necesario el estudio de las malezas que conviven con los cultivos en los campos paperos, de forma de determinar si actúan como reservorios para este fitopatógeno.

\section{Reconocimientos}

Agradecemos a los Ingenieros Agrónomos Alfonso Grela y Guillermo Galván por acompañarnos y colaborar en el muestreo, así como por su asesoramiento técnico junto al Ing. Agr. Enrique Verdier. Parte de este trabajo se realizó gracias al financiamiento del proyecto CSIC 273 sector productivo modalidad 2 (Universidad de la República).

\section{Referencias}

- ALEXANDER, E.; PHAM, D.; STECK, T. R. The viable-butnonculturable condition is induced by copper in Agrobacterium tumefaciens and Rhizobium leguminosarum. En: Applied and Environmental Microbiology. 1999, 65(8):3754-3756.

- ÁlvareZ, B.; BIOSCA, E. G.; LÓPEZ M. M. On the life of Ralstonia solanacearum, a destructive bacterial plant pathogen. En: MÉNDEZ-VILAS, A. Current research, technology and education topics in applied microbiology and microbial biotechnology. Vol. 1. Badajoz: Formatex, 2010. pp. 267-279

- CLSI. Interpretive criteria for identification of bacteria and fungi by DNA target sequencing; approved guideline. Wayne: CLSI, 2008. (CLSI document MM18-A)

- DENNY, T.P. Plant pathogenic Ralstonia species. En: GNANAMANICKAM, S.S. Plant associated bacteria. Dordrecht: Springer, 2006. pp. 573-644.

- DUMONTIER, Michel; HOGUE, Christopher W.V. NBLAST: a cluster variant of BLAST for $\mathrm{NxN}$ comparisons. En: $B M C$ Bioinformatics. 2002, 3:13

- ELPHINSTONE, J. G. Survival and possibilities for extinction of Pseudomonas solanacearum (Smith) Smith in cool climates. En: Potato Research. 1996, 39:403-410.

- ENGLEBRETCH, M.C. Modifications of a semi-selective medium for the isolation of Pseudomonas solanacearum. En: Bacterial Wilt Newsletter. 1994, 10:3-5.
- FEGAN, Mark; PRIOR, Philippe. How complex is the Ralstonia solanacearum specie complex? En: ALEN, C.; PRIOR, P.; HAYWARD, A. A. Bacterial wilt disease and the Ralstonia solanacearum specie complex. St. Paul: Amer Phytopathological Society, 2005. pp.437-445.

- GRANADA, G.A.; SEQUEIRA, L. Survival of Pseudomonas solanacearum in soil, rhizosphere and plant roots. En: Canadian Journal of Microbiology. 1983, 29:433-440.

- GREY, B. E.; STECK, T. R. The viable but nonculturable state of Ralstonia solanacearum may be involved in long-term survival and plant infection. En: Applied and Environmental Microbiology. 2001, 67:3866-3872.

- HAYWARD, A.C. Biology and epidemiology of bacterial wilt caused by Pseudomonas solanacearum. En: Annual Review of Phytopathology. 1991, 29:65-87.

- LIN, Chih-Hung; HSU, Shih-Tien; TZENG, Kuo-Ching; WANG, Jaw-Fen. Detection of race 1 strains of Ralstonia solanacearum in field samples in Taiwan using a BIO-PCR method. En: European Journal of Plant Pathology. 2009, 124(1):75-85.

- JANSE, J.D.; VAN DEN BELD, H.E.; ELPHINSTONE, J.; SIMPKINS, S.; TJOU-TAM-SIN, N.N.A.; VAN VAERENBERGH, J. Introduction to Europe of Ralstonia solanacearum biovar 2, race 3 in Pelargonium zonale cuttings. En: Journal of Plant Pathology. 2004, 86(2):147-155.

- KELMAN, A. The relationship of pathogenicity in Pseudomonas solanacerum to colony appearance on a tetrazolium medium. En: Phytopathology. 1954, 44:693-695.

- MCDOUGALD, D.; RICE, S. A.; WEICHART, D.; KJELLEBERG, S. Nonculturability: adaptation or debilitation? En: FEMS Microbiology Ecology. 1998, 25:1-9.

- MGAP. DIEA. Caracterización de la producción de papa. Encuesta 2003 [En línea]. Montevideo: MGAP, 2003, [Consulta: Junio 2012]. (Serie Encuestas; 214). Disponible en: http://www.mgap.gub.uy/ portal/agxppdwn.aspx?7,1,89,0,S,0,1498\%3bS\%3b1\%3b120,

- MGAP. Resolución ministerial 864/10, aprobación en las modificaciones al estándar especifico para producción de materiales de propagación de papa (Solanum tuberosum) de clase certificada, de 01 de noviembre de 2010. Montevideo: MGAP, 2010.

- MOFFETT, M.L.; GILES, J. E.; WOOD B. A. Survival of Pseudomonas solanacearum biovars 2 and 3 in soil: effect of moisture and soil type. En: Soil Biology and Biochemistry. 2002, 15(5):587-591.

- PASTRIK, K.H.; MAISS, E. Detection of Ralstonia solanacearum in potato tubers by polymerase chain reaction. En: Journal of Phytopathology. 2000, 148:619-626.

- PICARD, C.; PONSONNET, C.; PAGET, E.; NESME, X., SIMONET, P. Detection and enumeration of bacteria in soil by direct DNA extraction and polymerase chain reaction. En: Applied and Environmental Microbiology. 1992, 58:2717-2722.

- PRADHANANG, P.M.; ELPHINSTONE, J.G.; FOX R.T.V. Sensitive detection of Ralstonia solanacearum in soil: a comparison of different detection techniques. En: Plant Pathology. 2000, 49:414-422.

- SADDLER, G.S. Management of bacterial wilt disease. En: ALEN, C.; PRIOR, P.; HAYWARD, A. A. Bacterial wilt disease and the Ralstonia solanacearum specie complex. St. Paul: Amer Phytopathological Society, 2005. pp.121-132.

- SAMBROOK, J.; RUSSELL, D. Molecular cloning: a laboratory manual. 3ra ed. New York: Cold Spring Harbor, 2001.

- SANABRIA, A.; SIRI, M. I.; GRELA, A.; PIANZZOLA, M. J. Study of the persistence of Ralstonia solanacearum in soils 
focused to obtain a better advantage of the potato fields. En: 6th Latin American Biodeterioration and Biodegradation Symposium (Bogotá, mayo de 2007). Bogotá: [s.n.], 2007.

- SCHELL, Marc A. Control of virulence and pathogenicity genes of Ralstonia solanacearum by an elaborate sensory network. En: Annual Review of Phytopathology. 2000, 38:263-292.

- SEAL, S.E.; TAGHAVI, M.; FEGAN, N.; HAYWARD, A.C.; FEGAN, M. Determination of Ralstonia (Pseudomonas) solanacearum rDNA subgroups by PCR tests. En: Plant Patholology. 1999, 48:115-120.

- SIRI, M. I.; CECCHETTO, G.; ALDABE, L.; SANTOS, C.; SILVERA, E.; QUIRICI, L.; GALVÁN, G.; VILARÓ, F.; PIANZZOLA, M. J. Persistencia de Ralstonia solanacearum en chacras de Uruguay: diagnóstico molecular de muestras de suelo y susceptibilidad de Solanáceas nativas. En: SOCIEDAD URUGUAYA DE HORTIFRUTICULTURA. X Congreso Nacional de la Sociedad Uruguaya de Hortifruticultura, (Montevideo, 23-25 de mayo de 2005). Montevideo: Sociedad Uruguaya de Hortifruticultura, 2005.

- SIRI, M. I.; SANABRIA, A.; PIANZZOLA, M. J. Genetic diversity and aggressiveness of Ralstonia solanacearum strains causing bacterial wilt of potato in Uruguay. En: Plant Disease. 2011, 95(10):1292-1301.

- VAN ELSAS, J. D.; KASTELEIN, P.; VAN BEKKUM, P.; VAN DER WOLF, J. M., DE VRIES, P. M.; VAN OVERBEEK, L. S. Survival of Ralstonia solanacearum biovar 2, the causative agent of potato brown rot, in field and microcosm soils in temperate climates. En: Phytopathology. 2000, 90:1358-1366. 